Journal for ImmunoTherapy of Cancer

\section{Long-term survival of patients with stage III colon cancer treated with VRP-CEA(6D), an alphavirus vector that increases the CD8+ effector memory $T$ cell to Treg ratio}

To cite: Crosby EJ, Hobeika AC, Niedzwiecki D, et al. Long-term survival of patients with stage III colon cancer treated with VRP-CEA(6D), an alphavirus vector that increases the CD8+ effector memory $T$ cell to Treg ratio. Journal for ImmunoTherapy of Cancer 2020;8:e01662. doi:10.1136/ jitc-2020-001662

- Additional material is published online only. To view, please visit the journal online (http://dx.doi.org/10.1136/jitc2020-001662).

Accepted 20 0ctober 2020

Check for updates

(C) Author(s) (or their employer(s)) 2020. Re-use permitted under CC BY. Published by BMJ.

For numbered affiliations see end of article.

Correspondence to Dr Herbert Kim Lyerly; kim.lyerly@duke.edu

\section{ABSTRACT}

Background There remains a significant need to eliminate the risk of recurrence of resected cancers. Cancer vaccines are well tolerated and activate tumorspecific immune effectors and lead to long-term survival in some patients. We hypothesized that vaccination with alphaviral replicon particles encoding tumor associated antigens would generate clinically significant antitumor immunity to enable prolonged overall survival (OS) in patients with both metastatic and resected cancer. Methods OS was monitored for patients with stage IV cancer treated in a phase I study of virus-like replicon particle (VRP)-carcinoembryonic antigen (CEA), an alphaviral replicon particle encoding a modified CEA. An expansion cohort of patients $(n=12)$ with resected stage III colorectal cancer who had completed their standard postoperative adjuvant chemotherapy was administered VRP-CEA every 3 weeks for a total of 4 immunizations. OS and relapse-free survival (RFS) were determined, as well as preimmunization and postimmunization cellular and humoral immunity.

Results Among the patients with stage IV cancer, median follow-up was 10.9 years and 5-year survival was $17 \%$, (95\% Cl 6\% to 33\%). Among the patients with stage III cancer, the 5 -year RFS was $75 \%$, $(95 \% \mathrm{Cl} 40 \%$ to $91 \%)$; no deaths were observed. At a median follow-up of 5.8 years (range: $3.9-7.0$ years) all patients were still alive. All patients demonstrated CEA-specific humoral immunity. Patients with stage III cancer had an increase in CD8 $+\mathrm{T}_{\mathrm{EM}}$ (in 10/12) and decrease in F0XP3 + Tregs (in $10 / 12$ ) following vaccination. Further, CEA-specific, IFN $\gamma$ producing $C D 8+$ granzyme $B+T_{C M}$ cells were increased. Conclusions VRP-CEA induces antigen-specific effector $T$ cells while decreasing Tregs, suggesting favorable immune modulation. Long-term survivors were identified in both cohorts, suggesting the OS may be prolonged.

\section{BACKGROUND}

Colon cancer, while curable with surgery alone when localized to the bowel wall, has high rates of relapse when metastatic to lymph nodes (stage III) and high mortality rates when distantly metastatic (stage IV). ${ }^{1}$ Chemotherapy for stage III $^{2}{ }^{3}$ and chemotherapy plus biologic therapy with VEGF and EGFR-targeted antibodies in stage $\mathrm{IV}^{4}$ can both improve survival, but additional therapies are needed to extend these survival benefits.

Infiltration of colon cancers by $\mathrm{CD} 8+\mathrm{T}$ cells is associated with improved recurrence free survival, ${ }^{5}$ which suggests that attempts to augment the immune response against colon cancer may be beneficial. The success of immune checkpoint blockade (ICB) in metastatic microsatellite instability (MSI) high colorectal cancer ${ }^{6}$ has increased interest in immunotherapy for the remaining $85 \%-96 \%$ of patients with MSI; however, ICB alone has been ineffective in this subgroup. Mechanistically, the neoantigens generated by the genetic instability of MSI patients is thought to increase the number of potential tumor-specific effector $\mathrm{T}$ cells, which can be activated by ICB. Because more than $80 \%$ of colon cancer is MSI negative, ${ }^{7}$ alternative strategies to increase infiltrating T cells (TIL) and subsequent responses to ICB are needed.

Cancer vaccines that activate immune responses against tumor-expressed antigens, may be an option for increasing TIL and extending the efficacy of immunotherapy to colorectal cancer. ${ }^{8}$ Colon cancers express several defined antigens, relatively restricted to the tumor and known to be targets of immune effectors, with carcinoembryonic antigen (CEA) being one of the most extensively studied. ${ }^{9}$ Although studies frequently report induction of immune response against target antigens, clinical benefit associated 
with immunizations in the absence of ICB has been modest. ${ }^{10}$ Two potential explanations are the use of vaccines in heavily pretreated advanced cancer patients and immunization platforms that simultaneously activate immunosuppressive regulatory $\mathrm{T}$ cells along with the desired effector T cell response. ${ }^{11}$

Vaccine platforms for targeting CEA have included peptides, proteins, modified tumor cells, DNA, mRNA, viral vectors, and dendritic cells (DC). ${ }^{10}$ We previously reported on the use of virus-like replicon particle (VRP)CEA (AVX701), an alpha-VRP, based on attenuated Venezuelan equine encephalitis virus encoding the modified epitope CEA(6D) which we designate as VRP-CEA, to activate CEA-specific immune responses. ${ }^{12}$ CEA(6D) refers to an Asn to Asp substitution in CEA which results in enhanced recognition by cognate $\mathrm{CD} 8+\mathrm{T}$ cell receptors. ${ }^{13}$ Advantages of the VRP vaccine platform include their tropism for professional antigen-presenting cells (DCs), the capacity to replace their structural gene region with foreign genes, and the production of self-replicating RNA transcripts, resulting in generation of large amounts of the encoded heterologous proteins and induction of potent cellular and humoral immunity against these proteins. ${ }^{14}$ In the phase I clinical trial of VRP-CEA enrolling heavily pretreated patients with metastatic colorectal cancer, ${ }^{12}$ there was no dose limiting toxicity and the highest dose tested $\left(4 \times 10^{8} \mathrm{IU}\right)$ was determined to be the maximal feasible dose. CEA-specific $T$ cell and antibody responses following VRP-CEA vaccination were observed regardless of the development of antiviral neutralizing antibodies or regulatory $\mathrm{T}$ cell frequency.

We hypothesized that generating a high frequency of circulating CEA specific $\mathrm{T}$ cells generated, even with increased regulatory $\mathrm{T}$ cells, would provide some clinical benefit. Using a complex DC and pox vector based cancer vaccine, we have previously demonstrated improved survival of patients with metastatic colorectal cancer following metastasectomy and administration of the vaccine. ${ }^{15}$ These findings suggest that vaccination in a clinical scenario with minimal tumor-induced immunosuppression may be more effective, even without ICB. For these reasons, it has been suggested that patients following tumor resection with no evidence of residual disease but at a high risk of relapse may benefit from a vaccine that induces an adaptive immune response and could offer an improved overall survival (OS).

Consequently, we monitored the long-term survival of all patients with stage IV cancer who had been vaccinated with VRP-CEA. We observed three long-term overall survivors of over 10 years in patients with stage IV cancer that were vaccinated and underwent subsequent tumor debulking. We then enrolled an expansion cohort of patients with stage III colon cancer following surgery and completion of adjuvant systemic chemotherapy. At a median follow-up of 5.8 years (range: $3.9-7.0$ years), a $100 \%$ OS and $85 \%$ PFS among the patients with stage III colon cancer were vaccinated. Further, we identified increases in antigen-specific effector $\mathrm{T}$ cell responses and a reduction in Treg as early as after one vaccination.

\section{METHODS \\ Patients with stage III cancer and study drug administration}

All patients were enrolled and treated under an FDAapproved Investigational New Drug Exemption and registeredatClinicalTrials.gov (NCT00529984, NCT01890213). Participants were recruited from medical oncology clinics at Duke University Medical Center, Durham, North Carolina, USA and provided consent under protocols approved by the Duke University Medical Center Institutional Review Board (Pro00045976). Eligibility requirements for the stage IV study were previously reported. ${ }^{12}$ Eligibility requirements for the stage III study included age $\geq 18$, histologically confirmed stage III colon cancer as determined by the AJCC 7 th edition, and receipt of adjuvant postoperative chemotherapy (5-fluorouracil-based regimen with or without oxaliplatin for at least six cycles or capecitabine with or without oxaliplatin for four cycles). Chemotherapy must have been completed 1-6 months before initiating study treatment. Other requirements were ECOG status of 0 or 1 , adequate hematologic counts, and hepatic and renal function. Known autoimmune disease or HIV infection, concurrent immunosuppressive therapies, and significant cardiovascular disease or arrhythmias were exclusionary criteria.

VRP-CEA was administered at $4 \times 10^{8}$ IU intramuscularly into the deltoid (alternating arms with each injection) every 3 weeks for four administrations. Chest, abdominal, and pelvic CT or MRI scans and serum CEA level were performed as part of a patient's standard management at baseline and after the final vaccination. Blood samples were taken pretreatment, prior to each injection (weeks 0 , 3,6 , and 9), and 3 weeks post-treatment. Patients or their physicians were contacted approximately every 6 months for survival and progression status after completing study immunizations

\section{CYTOF flow cytometry analysis}

PBMC stimulation: Frozen vials were thawed and rested overnight at $37^{\circ} \mathrm{C}$. The timepoint postvaccination for each patient was chosen based on availability of PMBC samples (one patient at week 3 , three patients at week 6 , five patients at week 9 , and two patients at week 12). Cells were washed with warm RPMI-1640 medium (Hyclone) supplemented with $10 \%$ fetal bovine serum, (FBS) (Atlanta Biologicals), $100 \mathrm{U} / \mathrm{mL}$ penicillin, $100 \mu \mathrm{g}$ / $\mathrm{mL}$ streptomycin, $29.2 \mathrm{mg} / \mathrm{mL}$ L-glutamine (Hyclone) and $25 \mathrm{U} / \mathrm{mL}$ benzonase (Sigma-Aldrich) then resuspended at $5 \times 10^{6}$ cells $/ \mathrm{mL}$. Cells were restimulated with $\mathrm{BFA} /$ monensin $(1 \times$ both eBioscience) alone (negative control), BFA/monensin/PMA (500 ng/mL, Sigma)/ Ionomycin $(1 \mu \mathrm{g} / \mathrm{mL}$, Sigma) (positive control), or BFA/ monensin/TRICOM-CEA ${ }^{16}$ (10 MOI/cell; Lot\#MFG72299, a different vector expressing CEA to stimulated 
CEA but not VRP specific cells) for 5 hours prior to cytometry time of flight (CYTOF) staining.

CYTOF antibody panel (online supplemental table 1) lists the 28 labels with the corresponding antibodies. Antibodies were purchased from Fluidigm; those in gray were purchased from the Lederer lab at the Brigham and Women's Hospital; CTLA4-FITC was purchased from ThermoFisher. Concentration of each antibody was titrated and optimized individually.

Mass cytometry: Staining of samples was adapted from Protocol PN 400279 A4 (Fluidigm). Briefly, cells were then stained with Cell-ID Cisplatin at a final concentration of $1 \mu \mathrm{M}$ before FC blocking (TruStain FcX, Biolegend). Control PBMCs were stained with CD45-115In and experimental samples were stained with CD45-89Y in cell staining buffer (Fluidigm). Control PBMCs were spiked into each sample for a final proportion of $20 \%$ control cells: $80 \%$ experimental. Cells were stained with the remaining surface antibodies and washed into FoxP3-Fix/Perm buffer (eBioscience) at $4^{\circ} \mathrm{C}$ overnight. Samples were washed with FoxP3 Permwash and stained for intracellular antibodies. Cells were fixed in 1.6\% formaldehyde solution for 1 hour prior to Iridium intercalation in Maxpar Fix/Perm Buffer $(31.25 \mathrm{nM})$. Two additional water washes were performed immediately before running on the mass cytometer and samples were resuspended in 0.1X EQ four-element calibration beads (Fluidigm) at a concentration of $5 \times 10^{5} / \mathrm{mL}$. Samples were acquired on a Helios mass cytometer (Fluidigm) by the UNC mass cytometry core which is funded by the University Cancer Research Fund (UCRF) and UNC Cancer Center Core Support Grant \#P30CA016086.

CYTOF analysis: FCS files were uploaded to the Astrolabe Cytometry Platform (Astrolabe Diagnostics) where transformation, debarcoding, cleaning, labeling, and unsupervised clustering was done. FCS files were normalized through median bead intensity and beads were removed prior to analysis. Single-cell data have been clustered using the FlowSOMR package (RRID:SCR_016899) ${ }^{17}$ and labeled using the Ek'Balam algorithm. ${ }^{18}$ The MDS map was generated using the cmdscale $\mathrm{R}$ function. ${ }^{19}$ Differential abundance analysis was done using the edgeR R package (RRID:SCR_012802), ${ }^{20-22}$ differential expression analysis was done using the limma $\mathrm{R}$ package (RRID:SCR_010943), ${ }^{23}$ and cell subset definitions ${ }^{25}{ }^{26}$ follow published methods. The clusters were further analyzed using the Matthews Correlation Coefficient (MCC) to identify any additional changes in cytokine production. ${ }^{17}$ Cluster labeling, method implementation, differential abundance, differential expression, and visualization were done through the Astrolabe Cytometry Platform (Astrolabe Diagnostics, Inc.).

\section{Anti-CEA antibody response by ELISA}

Patient sera were collected at weeks $0,3,6,9$, and 12 . 96-well plates were coated with whole CEA protein $(100 \mathrm{ng} /$ well) and incubated with $100 \mu \mathrm{L}$ of serum in duplicate serially diluted $1: 25$ to 1:1600. Titers were defined as the highest dilution such that the mean absorbance was equal to twice the negative control.

\section{Analysis of antivector responses with a VRP neutralization assay}

To determine antivector responses, antibodies to VRP were measured using a modified neutralization assay previously described. ${ }^{27}$ VRP expressing HER2 was mixed with serial dilutions of patient sera and then added to Vero cells (RRID:CVCL_0059). The number of cells expressing HER2 for each serum dilution was determined by flow cytometry.

\section{Statistical analyses}

For clinical studies, descriptive statistics are presented. Relapse-free survival (RFS) was defined as the time from surgery to disease recurrence or death from any cause, whichever came first. For patients with stage III cancer, OS was defined from the time of surgery until last follow-up or death due to any cause. For patients with stage IV cancer, the starting date for OS was the date of study enrollment. RFS and OS were calculated using the Kaplan-Meier method. Radiographic response was determined according to RECIST criteria 1.1. A paired Student's t test was used to determine differences prevaccination and postvaccination. Data were analyzed using SAS software V.9.4 (Copyright 2016 SAS Institute; RRID:SCR_008567) and RStudio (R V.3.6.1).

\section{RESULTS \\ Long-term survival in patients with stage IV cancer treated with VRP-CEA(6D)}

In the prior clinical trial of VRP-CEA(6D) enrolling patients with metastatic malignancies (predominantly colon cancer), we observed vaccine-induced adaptive immunity and reported longer survival for those with CEA-specific $\mathrm{T}$ cell responses (details and demographics previously published). ${ }^{12}$ We now update their survival, with median follow-up of 10.9 years; $95 \%$ CI (9.6 to 11.4) with 10-year survival of $0.14 ; 95 \%$ CI $(0.04$ to 0.29$)$. Three of $28(3 / 28)$ patients were alive at $9.6,10.5$, and 11.4 years, respectively, from study enrollment (figure 1 ). These three individuals had previously treated metastatic cancer, but minimal or no evidence of disease at the time of enrollment, suggesting that activity of the vaccine may be greater in those with the least tumor-induced immunosuppression. We, therefore, designed a pilot study to assess the immunogenicity and clinical activity of VRPCEA(6D) in a group of patients with no evidence of disease but significant risk of recurrence, those with stage III colon cancer who had their primary disease resected and completed adjuvant chemotherapy.

\section{Patients with stage III cancer treated with VRP-CEA(6D)— patient demographics and treatment}

Patients ( $\mathrm{n}=12)$ with stage III colon cancer were subsequently enrolled onto this study at one center from 


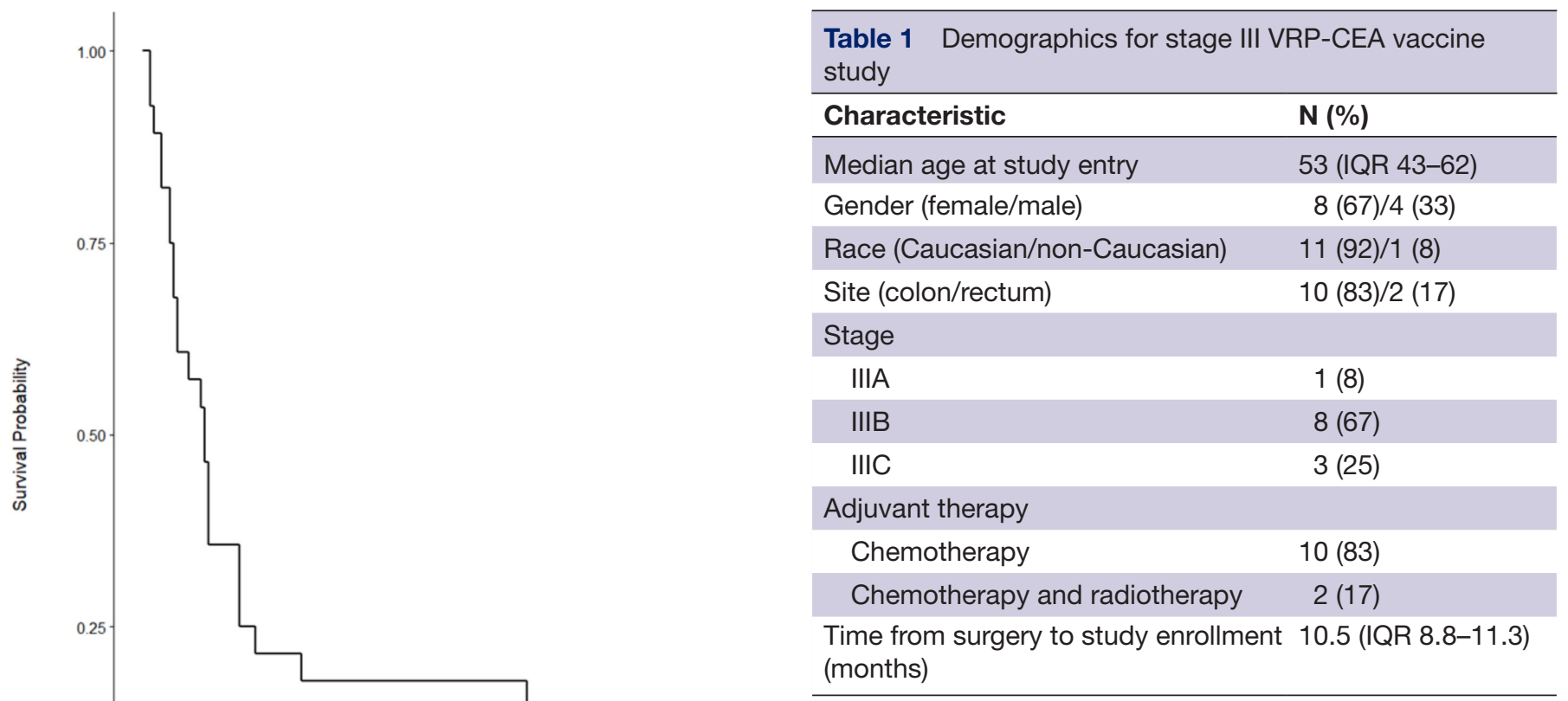

CEA, carcinoembryonic antigen; VRP, virus-like replicon particle.

tested postvaccination (online supplemental figure 1). The highest response postvaccine is shown for each patient. While this is a valuable tool, it is more difficult to determine small changes in antigen-specific $\mathrm{T}$ cell responsiveness and does not give information about $\mathrm{T}$ cell subsets responding to the vaccinations.

In order to characterize more comprehensively the induction of systemic $\mathrm{T}$ cell responses postvaccination, peripheral blood mononuclear cells (PBMCs) obtained before and after immunization were evaluated by multiparameter mass cytometry (CyTOF) following stimulation with a different CEA-encoding viral vector than used for vaccination (rF-CEA(6D)-TRICOM). The time postvaccination was dependent on PBMC availability, but all data are shown with paired prevaccine and postvaccine measurements for each patient. Cells were clustered using cell surface markers and expression of effector molecules prevaccination and postvaccination within these clusters was analyzed (figure 2A). Cell types were grouped into canonical subsets based on a gating hierarchy that corresponds to traditional gating used for flow cytometric analysis of surface markers. This process is automated to limit the introduction of bias that can occur when determining gates manually. To visualize the large amount of data that are generated by this CYTOF staining panel, we used a Multidimensional Scaling (MDS) map. Each bubble in this map represents a cell subset that was identified and analyzed and the size of that bubble is determined by the median frequency of cells contained in that bubble across all samples analyzed. We then compared the frequency of each identified cell type postvaccination to identify how these immune subsets were altered by the vaccine. Each bubble is colored based on the magnitude of fold change from the prevaccine to postvaccine sample after restimulation with TRICOM-CEA. A volcano plot summarizes the changes in subset frequency and the statistical 
A
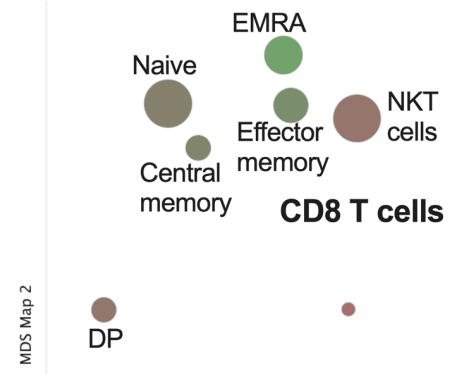

B

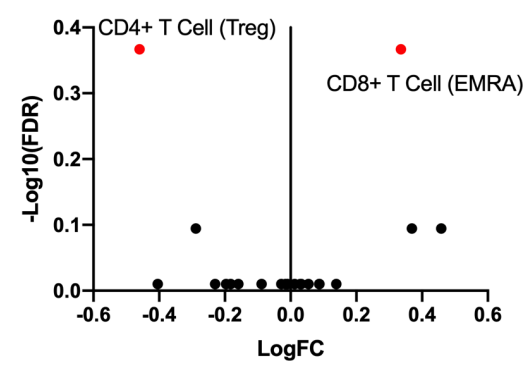

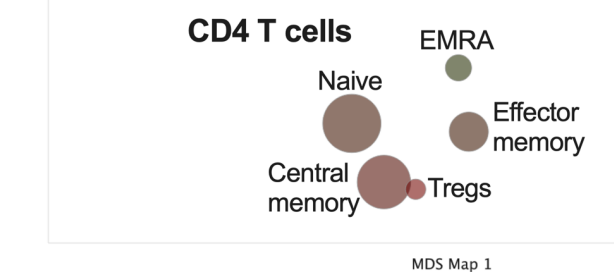
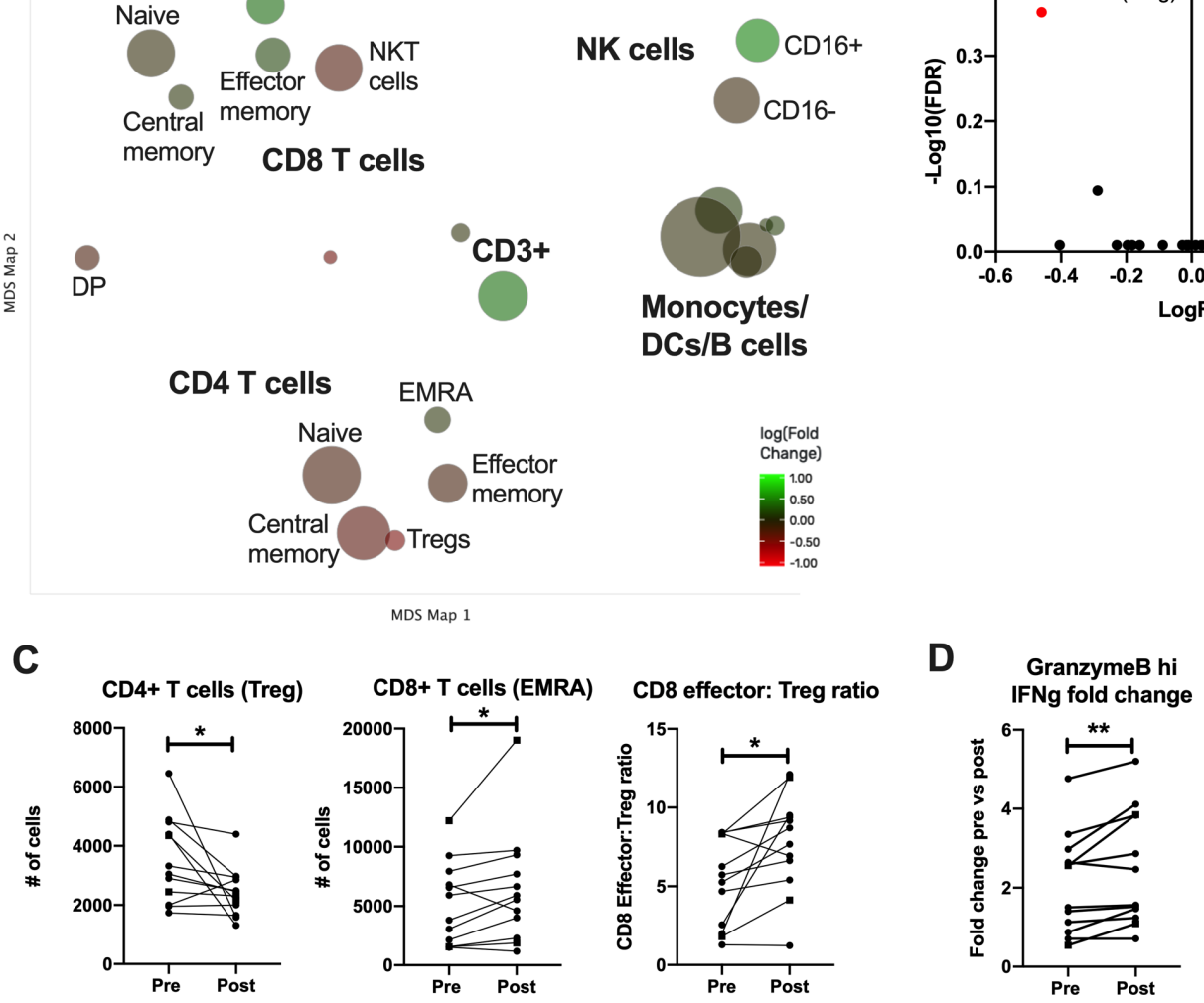

Figure 2 CYTOF analysis of PBMCs restimulated with CEA from patients prevaccination and postvaccination. (A) Cells were clustered and visualized using MDS. Each cluster is colored based on magnitude of fold change from the prevaccine to postvaccine sample after restimulation with TRICOM-CEA. (B) Volcano plot of identified cell clusters from (A), showing those with a significant $p$ value in red. (C) Number of cells in each indicated cluster or the ratio of activated CD8 T cells to Tregs paired for each patient prevaccination and postvaccination. Samples from patients with colorectal cancer are shown as dashed lines with square symbols. (D) The fold change in IFN- $\gamma$ production by granzyme B hi CD8 T cells post-TRICOM-CEA restimulation paired for each patient prevaccination and postvaccination. ${ }^{*} \mathrm{P}<0.05{ }^{*} \mathrm{p}<0.01$. CEA, carcinoembryonic antigen; CYTOF, cytometry time of flight; MDS, multidimensional scaling; PBMS, peripheral blood mononuclear cell; VRP, virus-like replicon particle.

significance of each change (figure 2B). We can see here that the only two cell subsets that are significantly changed postvaccination are CD8+ $\mathrm{T}_{\text {EMRA }}$, which increase postvaccination, and Tregs which decrease (shown in red). These changes are shown for each individual patient as well (figure 2C). We observed that $\mathrm{CD} 8+\mathrm{T}_{\mathrm{EM}}$ (and in particular the terminally differentiated effector memory cells CD8 $\mathrm{T}_{\text {EMRA }}$ ) were increased in 10/12 $(83.3 \%)$ patients and Tregs were decreased in 10/12 $(83.3 \%)$ patients following the immunizations, with both parameters changing in 8/12 (66.7\%) patients. Analysis of both parameters taken together shows that the CD8 $\mathrm{T}_{\mathrm{EMRA}}$ :Treg ratio increased in $10 / 12(83.3 \%)$ patients (figure 2C).

In addition to the cell surface marker based gating analysis, we used an unsupervised clustering algorithm to identify any subsets of all of the previously identified cell types that had statistically significant differential expression of cytokines following stimulation with CEA. As a result, we show that $\mathrm{CD} 8+$ granzyme $\mathrm{B}+\mathrm{T}_{\mathrm{CM}}$ cells generating IFN $\gamma$ were increased (in 9/12 (75\%)). No other cell type was significantly changed by immunization. These data indicate that an activated, cytolytic $\mathrm{T}$ cell population specific for CEA is induced by the VRP-CEA without resulting in an enhanced immunosuppressive population.

\section{Induction of antibody responses by VRP-CEA(6D)}

In our previous VRP-CEA study in patients with stage IV cancer, ${ }^{12}$ although viral replicon particle (VRP)-induced neutralizing antibodies were detected, we were nonetheless able to immunize repeatedly, increasing the humoral immune response. In the present study, neutralizing antibodies against VRP were induced in all but one patient after immunization (figure 3A). As before, despite these neutralizing antibodies, VRP-CEA activated CEAspecific antibodies with titers above baseline were noted in all patients (figure 3B). Consistent with our prior reports, these data suggest that the neutralizing antibodies, common to most viral vector platforms, do not impair VRP-CEA immunogenicity. As with the ELISPOT responses, the CEA specific antibody responses in patients with stage III cancer were significantly higher than those seen in patients with stage IV cancer. The anti-CEA titers in patients with stage IV cancer averaged a maximum titer 
A

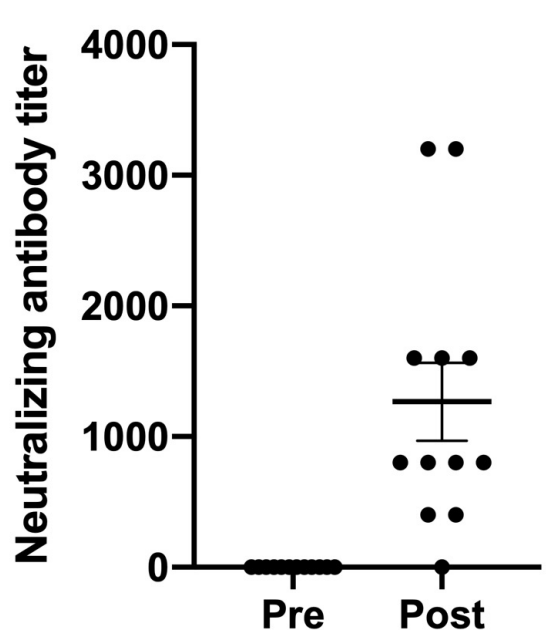

B

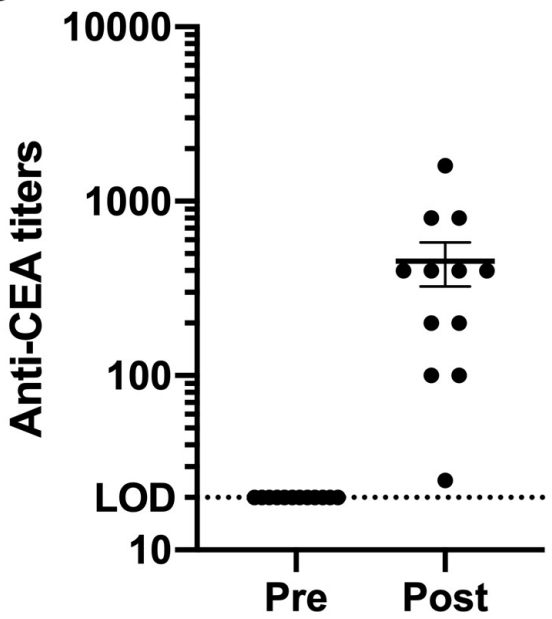

Figure 3 VPR neutralizing antibody titer before initiating immunizations and after immunizations were completed. (A) Patient sera were analyzed before and after VRP-CEA in anti-VRP neutralization assay. The endpoint titer was defined as the last serum dilution at which there was at least $80 \%$ reduction in the number of VRP positive cells compared with control wells. (B) Patient sera were analyzed for anti-VRP antibodies on weeks $0,3,6,9$, and 12. The antibody titer is presented for prevaccination and the highest postvaccination response. Values shown as mean \pm SEM. CEA, carcinoembryonic antigen; VRP, virus-like replicon particle.

of $80,{ }^{12}$ while patients with stage III cancer in this cohort had an average titer of nearly 500 (figure 3B).

\section{Clinical outcomes}

The clinical status of all participants was ascertained periodically following completion of treatment. At a median follow-up of 60 months (5.8 years), all patients remained alive and 3/12 (25\%) had recurrent disease (figure 4).

\section{DISCUSSION}

Many malignancies, despite resection and standard oncologic therapies, continue to have high risks of relapse. Stage III colon cancer, prior to the broad implementation of systemic therapy, was frequently lethal due to the emergence of recurrent disease in a majority of patients. The IDEA collaborative ${ }^{29}$ reported recently that the 5-year OS rate was $82.4 \%$ and $82.8 \%$ for 3 months and 6 months of postoperative adjuvant chemotherapy, respectively. The 5-year DFS rates were $69.1 \%$ and $70.8 \%$, respectively. This risk of recurrence in a scenario with minimal tumor burden to cause immunosuppression represents an ideal scenario for testing a cancer vaccine. Having demonstrated the immunogenicity of VRP-CEA in the presence of elevated levels of regulatory $\mathrm{T}$ cells in patients with advanced colorectal cancer, ${ }^{12}$ despite vector-induced neutralizing antibodies, we noted the long-term survival of a subset $(3 / 28,11 \%)$ of vaccinated patients with stage IV cancer. To determine if immune responses and clinical benefit would be enhanced in patients with less tumor burden, we performed this pilot study to assess the immunogenicity and estimate clinical results of VRP-CEA vaccination in patients with stage III colorectal cancer. The immunizations were well tolerated, an important point for a population of patients often left with chronic neuropathy from prior therapy and for whom the field is attempting to reduce the amount of therapy administered. At a median follow-up of 5.8 years, survival was $100 \%$ and only $3 / 12(25 \%)$ had experienced recurrent disease, both of which are not inconsistent with the outcome from the IDEA collaborative. ${ }^{29}$ Anti-CEA antibody levels above baseline were detected in all patients and the majority of patients experienced an increase in systemic CEA-specific $T$ cell responses, indicating a highly functional vaccine.

An important strength of this study was the use of CyTOF to measure the immune response in the peripheral blood following immunization. In our prior study with VRP-CEA in patients with stage IV cancer, we exclusively used ELISPOT analysis to characterize the $\mathrm{T}$ cell response; however, this provides a narrow description of immunogenicity and fails to completely capture the magnitude of $\mathrm{T}$ cells capable of responding to CEA before and after immunization. We recently reported on the use of CyTOF analysis to describe the changes occurring in peripheral blood immune cells after vaccination with a different VRP-based vaccine encoding the tumor antigen HER2 (VRP-HER2). ${ }^{28}$ In that study, we also observed the expansion of an antigen-specific, cytolytic CD8 T cell population, suggesting that CyTOF may be a preferred approach for assessing immunogenicity of cancer vaccines. In the current study, CyTOF allowed us to determine that the terminally differentiated effector memory cells CD8+ $\mathrm{T}_{\text {EMRA }}$ were increased and Tregs were decreased in most patients following the immunizations, with the result that the CD8 $\mathrm{T}_{\mathrm{EMRA}}$ : Treg ratio increased, an important indicator of a favorable environment for antitumor immune responses. Because our prior study with VRP-CEA did not use CyTOF analysis, we cannot directly 

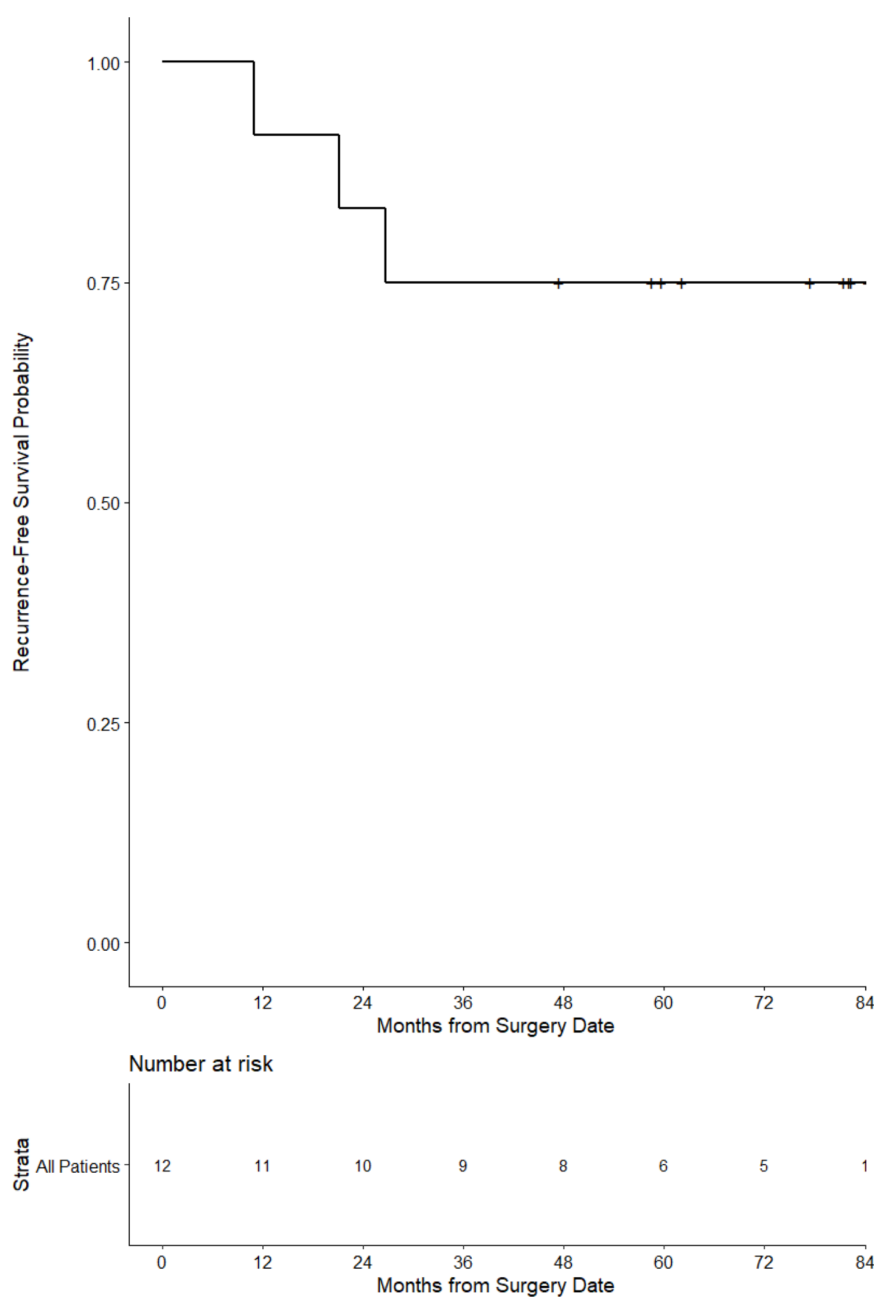

Figure 4 Progression-free survival for the stage III patients immunized with VRP-CEA. CEA, carcinoembryonic antigen; VRP, virus-like replicon particle.

compare the immunogenicity of this vector in patients with stage III versus stage IV colon cancer; however, we did perform an IFN $\gamma$-ELISPOT in a limited number of patients in the current study and observed much higher magnitudes of CEA-specific $\mathrm{T}$ cell responses compared with those seen in the stage IV study. ${ }^{12}$ The highest responses seen in patients with stage IV cancer averaged approximately 40 IFN- $\gamma$ producing cells per $10^{6}$ cells while patients with stage III cancer in this study had an average of approximately 150 IFN- $\gamma$ producing cells (online supplemental figure 1).

Because the OS and DFS of the vaccinated subjects were consistent with studies of patients who have received adjuvant therapy for stage III colon cancer, we were interested in determining whether any immune changes correlated with improved DFS. Due to the small number of recurrences, we were unable to make this assessment; however, the impact of immunotherapy may be greatest on OS which may take longer to demonstrate. In a clinical trial of a poxvector-based, CEA-targeting vaccine evaluated in patients with resected colorectal metastases, we found that OS but not recurrence-free survival was improved with the immunizations compared with unvaccinated patients. ${ }^{15}$ We will continue to follow participants in the current study to determine whether OS eventually does correlate with an immune biomarker.

Subsequent studies will attempt to enhance the potency of the induced $\mathrm{T}$ cell and antibody responses. One strategy could involve immunization in the neoadjuvant setting prior to surgical resection, a concept that has preliminarily demonstrated benefit for other forms of immunotherapy (eg, ICB) in other malignancies. ${ }^{30}{ }^{31}$ Another strategy could include a combination of the VRP-CEA with ICB. We recently reported that in mouse models of colon cancer, the combination of a viral-CEA vaccine with anti-PD-1 antibody resulted in greater antitumor activity and immune responses compared with vaccination against CEA alone. ${ }^{32}$

We conclude that immunization of patients with colon cancer with a viral replicon-based cancer vaccine induces antigen-specific effector $\mathrm{T}$ cells while decreasing Tregs, a feature of effective antitumor immune responses. Patients with minimal residual disease achieved surgically appear to have the greatest benefit from this vaccine. Future studies will test novel dosing schedules and combinations with ICB.

\section{Author affiliations}

${ }^{1}$ Surgery, Duke University School of Medicine, Durham, North Carolina, USA ${ }^{2}$ Biostatistics and Bioinformatics, Duke University School of Medicine, Durham, North Carolina, USA

${ }^{3}$ Biostatistics, Duke Cancer Institute, Durham, North Carolina, USA

${ }^{4}$ Medicine, Duke University School of Medicine, Durham, North Carolina, USA

${ }^{5}$ HDT Bio Corp, Seattle, Washington, USA

${ }^{6}$ VLP Therapeutics, Gaithersburg, Maryland, USA

${ }^{7}$ Medicine, University of Washington, Seattle, Washington, USA

${ }^{8}$ Pathology, Duke University School of Medicine, Durham, North Carolina, USA

${ }^{9}$ Immunology, Duke University School of Medicine, Durham, North Carolina, USA

Acknowledgements The authors wish to thank Delila Serra, Amanda Bradshaw, Karrie Comatas, and Wiguins Etienne for their performance of the immune monitoring assays including neutralization assays; Sherri Haley and Wanda Honeycutt for their nursing management of the patients on the clinical trial; Katherine Kalinowski for patient outcomes ascertainment; Dr Bolyn Hubby and Whitney Lewis for the molecular construction and characterization of the VRP vectors including VRP-CEA. The authors would like to acknowledge Tao Wang for her technical assistance with acquiring flow cytometry data. The authors would like to acknowledge the University of North Carolina Mass Cytometry Core, University Cancer Research Fund (UCRF), and UNC Cancer Center Core Support Grant \#P30CA016086 for acquisition of the CYTOF samples.

Contributors EJC conceived and designed the analysis, collected the data, performed the analysis, wrote the manuscript, and edited the manuscript. AH conceived and designed the analysis, collected the data, oversaw the project, and edited the manuscript. DN performed the analysis and edited the manuscript. CR performed the analysis and edited the manuscript. DH collected the data and edited the manuscript. PB conceived and designed the analysis, edited the manuscript, and provided funding. JS conceived and designed the analysis, edited the manuscript, and provided funding. WG collected the data and edited the manuscript. TO collected the data, performed the analysis, and edited the manuscript. MAM conceived and designed the analysis, collected the data, oversaw the project, wrote the manuscript, edited the manuscript, and provided funding. ZCH collected the data, performed the analysis, and edited the manuscript. HKL conceived and designed the analysis, collected the data, oversaw the project, edited the manuscript, and provided funding.

Funding This work was supported by a clinical trial grant from AlphaVax Human Vaccines (PI: Michael Morse), a grant from the NIH National Cancer Institute (principal investigator: H.K. Lyerly [NCI P01-CA078673]), and the George Barth Gellar Professorship (H.K. Lyerly). 
Competing interests PB and JS were employed by AlphaVax Inc. HKL was on the Scientific Advisory Board of AlphaVax Inc and has equity in AlphaVax.

\section{Patient consent for publication Not required.}

Ethics approval All protocols were approved by the Duke University Medical Center Institutional Review Board (Pro00045976) and participants gave informed consent before taking part.

\section{Provenance and peer review Not commissioned; externally peer reviewed.}

Data availability statement Data sharing not applicable as no datasets generated and/or analyzed for this study. Raw files from CTYOF analysis are available on request.

Supplemental material This content has been supplied by the author(s). It has not been vetted by BMJ Publishing Group Limited (BMJ) and may not have been peer-reviewed. Any opinions or recommendations discussed are solely those of the author(s) and are not endorsed by BMJ. BMJ disclaims all liability and responsibility arising from any reliance placed on the content. Where the content includes any translated material, BMJ does not warrant the accuracy and reliability of the translations (including but not limited to local regulations, clinical guidelines, terminology, drug names and drug dosages), and is not responsible for any error and/or omissions arising from translation and adaptation or otherwise.

Open access This is an open access article distributed in accordance with the Creative Commons Attribution 4.0 Unported (CC BY 4.0) license, which permits others to copy, redistribute, remix, transform and build upon this work for any purpose, provided the original work is properly cited, a link to the licence is given, and indication of whether changes were made. See https://creativecommons.org/ licenses/by/4.0/.

\section{ORCID iDs}

Erika J Crosby http://orcid.org/0000-0002-4872-6711

Zachary C Hartman http://orcid.org/0000-0001-6549-8207

Herbert Kim Lyerly http://orcid.org/0000-0002-0063-4770

\section{REFERENCES}

1 Survival rates for colorectal cancer: American cancer Society, 2020. Available: https://www.cancer.org/cancer/colon-rectal-cancer/ detection-diagnosis-staging/survival-rates.html [Accessed 20 Jun 2020].

2 André T, de Gramont A, Vernerey D, et al. Adjuvant Fluorouracil, Leucovorin, and Oxaliplatin in Stage II to III Colon Cancer: Updated 10-Year Survival and Outcomes According to BRAF Mutation and Mismatch Repair Status of the MOSAIC Study. J Clin Oncol 2015;33:4176-87.

3 Grothey A, Sobrero AF, Shields AF, et al. Duration of adjuvant chemotherapy for stage III colon cancer. N Engl J Med Overseas Ed 2018;378:1177-88.

4 Venook AP, Niedzwiecki D, Lenz H-J, et al. Effect of first-line chemotherapy combined with cetuximab or bevacizumab on overall survival in patients with KRAS wild-type advanced or metastatic colorectal cancer: a randomized clinical trial. JAMA 2017;317:2392-401.

5 Pagès $F$, Berger $A$, Camus $M$, et al. Effector memory $T$ cells, early metastasis, and survival in colorectal cancer. $N$ Engl $J$ Med 2005;353:2654-66.

6 Morse MA, Hochster H, Benson A, Al B. Perspectives on treatment of metastatic colorectal cancer with immune checkpoint inhibitor therapy. Oncologist 2020;25:33-45.

7 Battaglin F, Naseem M, Lenz H-J, et al. Microsatellite instability in colorectal cancer: overview of its clinical significance and novel perspectives. Clin Adv Hematol Oncol 2018;16:735-47.

8 Merika E, Saif MW, Katz A, et al. Review. colon cancer vaccines: an update. In Vivo 2010;24:607-28.

9 Bashir B, Snook AE. Immunotherapy regimens for metastatic colorectal carcinomas. Hum Vaccin Immunother 2018;14:250-4.

10 Berry J, Vreeland T, Trappey A, et al. Cancer vaccines in colon and rectal cancer over the last decade: lessons learned and future directions. Expert Rev Clin Immunol 2017;13:235-45.
11 Quandt J, Schlude C, Bartoschek M, et al. Long-peptide vaccination with driver gene mutations in p53 and KRAS induces cancer mutation-specific effector as well as regulatory $T$ cell responses. Oncoimmunology 2018;7:e1500671.

12 Morse MA, Hobeika AC, Osada T, et al. An alphavirus vector overcomes the presence of neutralizing antibodies and elevated numbers of Tregs to induce immune responses in humans with advanced cancer. J Clin Invest 2010;120:3234-41.

13 Zaremba S, Barzaga E, Zhu M, et al. Identification of an enhancer agonist cytotoxic T lymphocyte peptide from human carcinoembryonic antigen. Cancer Res 1997;57:4570-7.

14 Fuller DH, Berglund P. Amplifying RNA vaccine development. N Engl $J$ Med 2020;382:2469-71.

15 Morse MA, Niedzwiecki D, Marshall JL, et al. A randomized phase II study of immunization with dendritic cells modified with poxvectors encoding CEA and MUC1 compared with the same poxvectors plus GM-CSF for resected metastatic colorectal cancer. Ann Surg 2013;258:879-86.

16 Morse MA. Technology evaluation: CEA-TRICOM, Therion biologics Corp. Curr Opin Mol Ther 2001;3:407-12.

17 Van Gassen S, Callebaut B, Van Helden MJ, et al. FlowSOM: using self-organizing maps for visualization and interpretation of cytometry data. Cytometry A 2015;87:636-45.

18 Amir E-AD, Lee B, Badoual P, et al. Development of a comprehensive antibody staining database using a standardized analytics pipeline. Front Immunol 2019;10:1315.

19 Torgerson W. Theory and methods of scaling - torgerson, ws, 1958.

20 Robinson MD, McCarthy DJ, Smyth GK. edgeR: a Bioconductor package for differential expression analysis of digital gene expression data. Bioinformatics 2010;26:139-40.

21 McCarthy DJ, Chen Y, Smyth GK. Differential expression analysis of multifactor RNA-seq experiments with respect to biological variation. Nucleic Acids Res 2012;40:4288-97.

22 Lun ATL, Richard AC, Marioni JC. Testing for differential abundance in mass cytometry data. Nat Methods 2017;14:707-709.

23 Ritchie ME, Phipson B, Wu D, et al. limma powers differential expression analyses for RNA-sequencing and microarray studies. Nucleic Acids Res 2015;43:e47.

24 Weber LM, Nowicka M, Soneson C, et al. diffcyt: differential discovery in high-dimensional cytometry via high-resolution clustering. Commun Biol 2019;2:183.

25 Maecker HT, McCoy JP, Nussenblatt R. Standardizing immunophenotyping for the human immunology project. Nat Rev Immunol 2012;12:191-200.

26 Finak G, Langweiler M, Jaimes M, et al. Standardizing flow cytometry immunophenotyping analysis from the human immunophenotyping Consortium. Sci Rep 2016;6.

27 Bernstein DI, Reap EA, Katen K, et al. Randomized, doubleblind, phase 1 trial of an alphavirus replicon vaccine for cytomegalovirus in CMV seronegative adult volunteers. Vaccine 2009;28:484-93.

28 Crosby EJ, Gwin W, Blackwell K, et al. Vaccine-Induced Memory $\mathrm{CD}^{+}$T Cells Provide Clinical Benefit in HER2 Expressing Breast Cancer: A Mouse to Human Translational Study. Clin Cancer Res 2019;25:2725-36.

29 Sobrero AF, Andre T, Meyerhardt JA, et al. Overall survival (OS) and long-term disease-free survival (DFS) of three versus six months of adjuvant (adj) oxaliplatin and fluoropyrimidine-based therapy for patients (PTS) with stage III colon cancer (CC): final results from the idea (International duration evaluation of Adj chemotherapy) collaboration. JCO 2020;38:4004-04.

30 Blank CU, Rozeman EA, Fanchi LF, et al. Neoadjuvant versus adjuvant ipilimumab plus nivolumab in macroscopic stage III melanoma. Nat Med 2018;24:1655-61.

31 Blank CU, Versluis JM, Rozeman EA, et al. Abstract 3412: 36-months and 18-months relapse-free survival after (neo) adjuvant ipilimumab plus nivolumab in macroscopic stage III melanoma patients - update of the OpACIN and OpACIN-neo trials. Cancer Res 2020;80:3412-12.

32 Sun Y, Wang S, Yang H, et al. Impact of synchronized anti-PD-1 with Ad-CEA vaccination on inhibition of colon cancer growth. Immunotherapy 2019;11:953-66. 